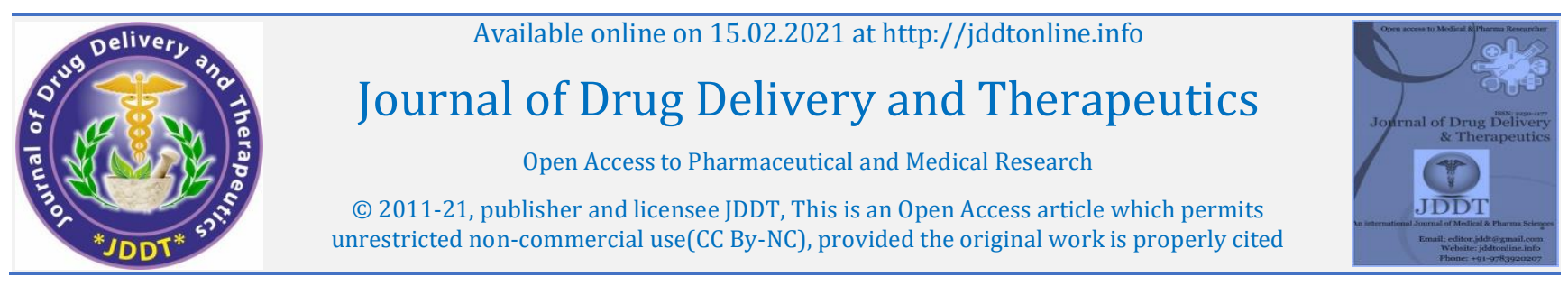

Open Access Full Text Article

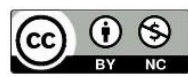

Research Article

\title{
Formulation development and characterization of in-situ gel of Rizatriptan Benzoate for intranasal delivery
}

\author{
Jalaram H. Thakkar*1, Shailesh T. Prajapati² \\ 1 Department of Pharmaceutical Sciences, Hemchandracharya North Gujarat University, Patan, Gujarat 384265, India \\ ${ }^{2}$ Kalol Institute of Pharmacy, Gujarat Technological University, Kalol, Gujarat 382721, India
}

Article Info:

Article History:

Received 04 Dec 2020;

Review Completed 10 Jan 2021

Accepted 19 Jan 2021;

Available online 15 Feb 2021

\section{Cite this article as:}

Thakkar JH, Prajapati ST, Formulation development and characterization of in-situ gel of Rizatriptan Benzoate for intranasal delivery, Journal of Drug Delivery and Therapeutics. 2021; 11(1-s):1-6

DOI: http://dx.doi.org/10.22270/jddt.v11i1-s.4685

\section{Abstract}

The present investigation was aimed to formulate and characterize ion-activated in-situ gel loaded with Rizatriptan Benzoate (RIZ) for intranasal administration for brain targeting. The gel was further optimized for process and formulation parameters by using 32 factorial design. The optimized batch having the concentrations of gellan gum and HPMC E15 LV $33.83 \mathrm{mg}$ and $9.6 \mathrm{mg}$ respectively. Gel strength and mucoadhesive strength of the optimized formulation were found to be $32.54 \mathrm{sec}$ and 2580.50 dynes/cm2 respectively. Moreover, improved in-vitro and ex-vivo release profile of insitu gel were observed in comparison to drug solution. In a nutshell, the developed formulation holds a great promise in overcoming the limitation associated with currently marketed RIZ formulations and illustrates the potential use of ion-activated in-situ gel to administer the drug by nasal route for brain targeting.

Keywords: In-situ gel, Rizatriptan benzoate, Ion-activated, Gellan gum, HPMC E15 LV, Brain delivery, Migraine.

\footnotetext{
*Address for Correspondence:

Jalaram H. Thakkar, Department of Pharmaceutical Sciences, Hemchandracharya North Gujarat University, Patan, Gujarat 384265, India.
}

\section{INTRODUCTION}

The nasal route has been potentially explored as an alternative route for the administration of vaccines and bio molecules such as proteins, peptides and non-peptide drugs that are susceptible to enzymatic or acidic degradation and first-pass hepatic metabolism. The nasal mucosa is one of the most permeable and highly vascularized sites for drug administration ensuring rapid absorption and onset of therapeutic action. Many drugs are not effectively and efficiently delivered to the brain using conventional drug delivery approaches. Transport of drugs from systemic circulation into the central nervous system (CNS) is restricted by the BBB and blood-CSF barrier 1,2 . While the BBB serves to protect the brain spinal cord from a variety of pathogens and toxic substances, it also presents a significant barrier to treating CNS disorders. Mainly charged, hydrophilic, water soluble substances, and large therapeutic agents are inhibited or prevented from entering the brain by the BBB ${ }^{3}$. Innovation is a key driver of growth that in the recent years there has been a continuous effort in the direction of achieving controlled and sustained drug delivery systems. Considerable attention has been received in the in-situ gelling systems over the past few years 4 . In situ gel formulation is executed for targeted delivery through the vaginal and rectal routes, and the nasal mucosa, circumventing the hepatic first pass metabolism ${ }^{5-9}$. Different mechanisms exist which provoke the formulation of in situ gels, such as those based on physiologic stimuli (e.g. temperature modifications, $\mathrm{pH}$ - triggered systems), those based on physical changes in biomaterials (e.g. solvent exchange and swelling), and those based on chemical reactions (e.g. UV radiation, ionic crosslinking, and ion-activated systems) ${ }^{10,11 .}$

Nasal route offers the advantage of controlled drug delivery for extended periods of time. The success of a controlled release product is directly linked to patient compliance which in situ gels can offer. Exploitation of polymeric in situ nasal gels for controlled release of drug provides numerous advantages over conventional dosage forms and can be considered as reliable and non-invasive drug delivery system. Exploration of novel gel triggering mechanisms and use of water-soluble, biodegradable polymers for product development of the in situ nasal gel formulations makes them more acceptable 12, 13. Thus, targeting the drug to the brain would decrease its distribution to other organs and thereby reduce the side effects. It would also result in lowering of dose and related toxicity.

Rizatriptan is a 5-HT1 agonist triptan drug used in the treatment of migraine headaches. Rizatriptan has mean oral absolute bioavailability of about $45 \%$, and mean plasma half- 
life of 2-3 hrs. Thus, the present investigation was aimed to formulate in-situ gelling system of Rizatriptan Benzoate targeted to brain via intranasal instillation which overcomes the above problems such as low bioavailability due to firstpass metabolism, adverse effects etc. and furthermore provide quick onset of action along with prolonged release of drug.

\section{MATERIALS AND METHODS}

\subsection{Materials}

Rizatriptan Benzoate (RIZ) was obtained as a gift sample from Dr. Reddy's Lab, Hyderabad, India. HPMC E15 LV and Gellan gum were purchased from Sigma Aldrich. Methanol HPLC grade, Acetonitrile HPLC grade and ortho-phosphoric acid (OPA) HPLC grade were purchased from Spectrochem Pvt. Ltd., Mumbai, India. All other reagents of analytical grade (AR) were used in the study.

\subsection{Methods}

\subsubsection{Preparation and optimization of Rizatriptan Benzoate in-situ gelling system}

Briefly, Gellan gum and HPMC E15 LV were dissolved in $5 \mathrm{ml}$ WFI and heated upto $90{ }^{\circ} \mathrm{C}$ for $20 \mathrm{~min}$ with continuous stirring using magnetic stirrer at $400-500 \mathrm{rpm}$. In another beaker, Drug was dissolved in $5 \mathrm{ml}$ WFI. Then the drug solution was added dropwise into the polymeric solution. The transparent solution was formed $\mathrm{pH}$ was adjusted in the range of 4.5 to 6.5. Final formulation was found to be transparent liquid solution. The development of in-situ gelling system can be made more efficient by using experimental design. In this approach, multiple variables that may affect the formulation properties are changed simultaneously in a strategic way, resulting in fewer experiments, interactions between variables being identified and quantified by the response parameters for the optimization of the formulation. Rizatriptan Benzoate in-situ gelling system was formulated by using $3^{2}$ full factorial design (Design Expert 7.0 Software). Here, gellan gum (30$40 \mathrm{mg}$ ) (A) and HPMC E15 LV (5-15 mg) (B) were selected as the independent variables and \% Drug release (Y1), Gel strength (Y2), and Mucoadhesive strength (Y3) as dependent variables. Statistical analysis was also carried out by the design expert software 7.0.3 to understand the response coefficient significance and interaction of the independent factors.

\subsubsection{Characterization}

\subsubsection{Gel strength}

In situ gel formulation (50 g) was placed in a $100 \mathrm{ml}$ measuring cylinder and gelation was induced by simulated nasal fluid. In each test-tube, a plunger was placed upon which $30 \mathrm{~g}$ weight was placed on the gel. The gel strength was determined by the time in seconds required by the weight to penetrate $5 \mathrm{~cm}$ deep into the gel.

\subsubsection{Mucoadhesive strength}

Freshly excised goat nasal mucosa (obtained from Alka Mutton Shop, Vadodara, India) was dipped immediately in phosphate buffer $\mathrm{pH}$ 7.4. Cartilages were removed properly, and the mucosal membrane was isolated and washed with phosphate buffer $\mathrm{pH}$ 7.4. Mucoadhesive strength was determined by using texture analyzer. $50 \mathrm{mg}$ of gel was placed on mucosal surface. The glass mounted mucosal surface with gel formulation and mucosal surface attached to cylinder were held in contact with each other for 2 min to ensure intimate contact between them. The mucoadhesive force expressed as the detachment stress in dynes $/ \mathrm{cm}^{2}$ was determined from the minimal weight that detached the mucosal tissue from surface of each formulation ${ }^{6}$.

$$
\text { Mucoadhesive strength (dynes } / \mathrm{cm} 2)=\mathrm{mg} / \mathrm{A}
$$

Where,

$\mathrm{m}=$ Weight required for detachment in gram, $\mathrm{g}=$ Acceleration due to gravity $(980 \mathrm{~cm} / \mathrm{s} 2), \mathrm{A}=$ Area of mucosa exposed.

\subsubsection{In-vitro drug release study}

In-vitro release study of RIZ loaded in-situ gel was carried out by using dialysis bag diffusion technique 14 . The drug release study was performed using $\mathrm{pH} 7.4$ phosphate buffered saline (PBS). The drug release for Rizatriptan benzoate solution and Rizatriptan benzoate loaded in-situ gel were determined. The gel equivalents to $2 \mathrm{mg}$ of Rizatriptan benzoate were placed in a dialysis bag, which was previously dipped overnight in diffusion medium, cleaned and sealed at both ends. The dialysis bag was immersed in the receptor compartment containing $30 \mathrm{ml}$ of pH 7.4 PBS, which was stirred at $100 \mathrm{rpm}$ and maintained at $37 \pm 2^{\circ} \mathrm{C}$. The receptor compartment was covered with aluminum foil to prevent the evaporation of release medium. Samples $(1 \mathrm{ml})$ were withdrawn at regular time intervals from the receptor compartment and the same volume $(1 \mathrm{ml})$ was replaced by fresh diffusion medium. The samples were analyzed using UV-Visible Spectrophotometer at $225 \mathrm{~nm}$. Rizatriptan benzoate solution in distilled water $(2 \mathrm{mg} / \mathrm{ml})$ was also evaluated for in-vitro drug release study 6.

\subsubsection{Ex-vivo drug permeability study}

Freshly excised goat nasal mucosa was collected from the slaughter house and dipped immediately in PBS pH 7.4. The excised superior nasal membrane was then mounted on Franz diffusion cell. The tissue was stabilized using phosphate buffer PH 7.4 in both the compartments (donor and receptor) and stirred for $15 \mathrm{~min}$ using a magnetic stirrer. After $15 \mathrm{~min}$, solution from both the compartments was removed and fresh PBS PH 7.4 was filled in the acceptor compartment. The temperature of receiver chamber, containing $8 \mathrm{ml}$ of diffusion media was controlled at $37 \pm 2^{\circ} \mathrm{C}$ under continuous stirring using teflon coated small magnetic bead at a constant rate $(100 \mathrm{rpm})$, in a way that the nasal membrane surface just in contact with the diffusion fluid. At the predetermined time intervals $(0,1,2,3,4,5,6,7 \ldots 28 \mathrm{hr})$, samples $(1 \mathrm{ml})$ from the receptor compartment were withdrawn and replaced by an equal volume of PBS pH 7.4. The samples were analyzed using UV-Visible Spectrophotometer at $225 \mathrm{~nm} \mathrm{15,16.}$

\subsubsection{Stability study}

Stability study of the optimized batch of RIZ loaded in-situ gel was conducted at different storage conditions for 2 months as per the ICH guidelines. The optimized batch was then characterized for $\mathrm{pH}$, gelation time and drug content at the time interval of 15 days, 30 days and 60 days ${ }^{17-20}$.

\section{RESULTS AND DISCUSSION}

\subsection{Preparation and optimization of RIZ loaded in-situ gel}

The selected levels of gellan gum and HPMC E15 LV incorporated into a 32 full factorial design and different formulations were prepared. $3^{2}$ full factorial design is an effective technique of RSM (response surface methodology) for optimizing complex process. The experimental design and statistical analysis were performed using design expert software. In this design total amount of the components were kept constant, 
while the proportion of the components was changed in the design. The independent variables are proportion of concentration of gellan gum (mg) and HPMC E15 LV (mg), while dependent variables were Drug release (\%), Gel strength (sec) and Mucoadhesive strength (dynes/ $\mathrm{cm}^{2}$ ). Total of 9 experiments were designed by the software shown in Table 1. From the results, optimized formulation parameters like concentration of gellan gum and HPMC E15 LV were found to be $33.83 \mathrm{mg}$ and $9.6 \mathrm{mg}$ respectively. Total 17 runs with 5 centre points were obtained from the BBD design matrix and the results are shown in table 1.

Table 1 Optimization of Rizatriptan Benzoate Loaded in-situ gel

\begin{tabular}{|c|c|c|c|c|c|}
\hline \multirow[b]{2}{*}{ Runs } & \multicolumn{2}{|c|}{ Actual value } & \multicolumn{3}{|c|}{ Response Parameters } \\
\hline & $\begin{array}{l}\text { Concentration } \\
\text { of G ellan gum } \\
\text { (X1) (mg) }\end{array}$ & $\begin{array}{c}\text { Concentration of } \\
\text { HPMC E15 LV(X2) } \\
\text { (mg) }\end{array}$ & $\begin{array}{c}\text { Y1 } \\
(\% \text { Drug } \\
\text { release at } 6 \mathrm{hr})\end{array}$ & $\begin{array}{l}\text { Y2 } \\
\text { (Gel Strength } \\
\text { (sec) }\end{array}$ & $\begin{array}{c}\text { Y3 } \\
\text { (Mucoadhesive } \\
\text { strength (dynes/cm²) }\end{array}$ \\
\hline 1 & 30 & 5 & 96.88 & 19.67 & 2165 \\
\hline 2 & 40 & 5 & 82.8 & 44.33 & 2567 \\
\hline 3 & 40 & 15 & 73.92 & 54.00 & 3105 \\
\hline 4 & 30 & 15 & 71.23 & 27.33 & 2723 \\
\hline 5 & 35 & 15 & 78.84 & 40.67 & 2912 \\
\hline 6 & 35 & 10 & 82.8 & 32.33 & 2589 \\
\hline 7 & 40 & 10 & 80.94 & 52.67 & 2879 \\
\hline 8 & 30 & 10 & 74.51 & 21.02 & 2501 \\
\hline 9 & 35 & 5 & 96.19 & 33.01 & 2387 \\
\hline
\end{tabular}

\subsubsection{Statistical analysis}

The values of the dependent variables \% Drug release (Y1), Gel strength (Y2), and Mucoadhesive strength (Y3) were incorporated into the design expert software 7.3.3 and to evaluate the effect of the formulation variables on the response variables, polynomial equations were generated using design expert software 7.3.3.

$\%$ Drug release $=82.0-0.82 \mathrm{X} 1-8.6 \mathrm{X}_{2}$
Gel strength $=36.10+13.85 \mathrm{X}_{1}+4.20 \mathrm{X}_{2}$

Mucoadhesive strength $=2651.20+190.61 \mathrm{X}_{1}+26.49 \mathrm{X}_{2}$

The interaction between the various combinations of the independent variables that can affect the response parameters were studied and are represented graphically as counter plots (figure 1).

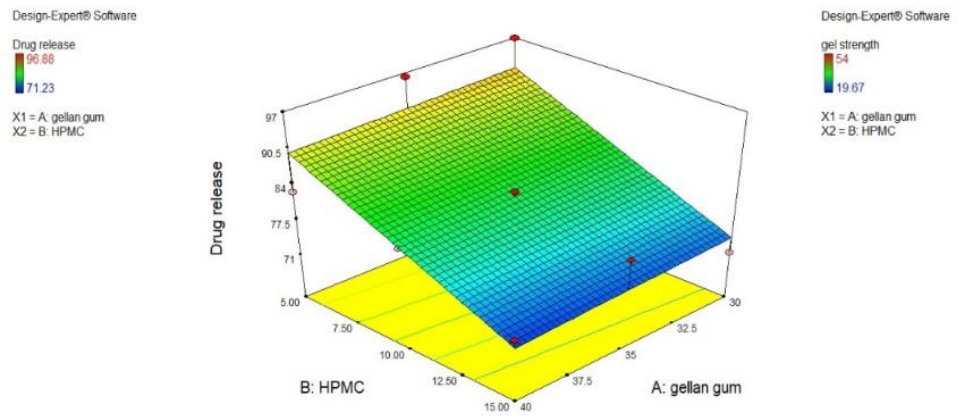

A) Counter plot of Drug release

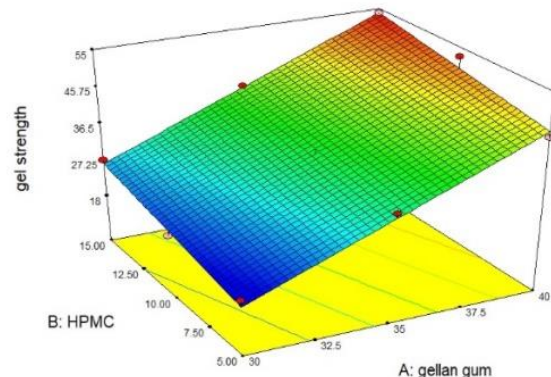

B) Counter plot of Gel strength

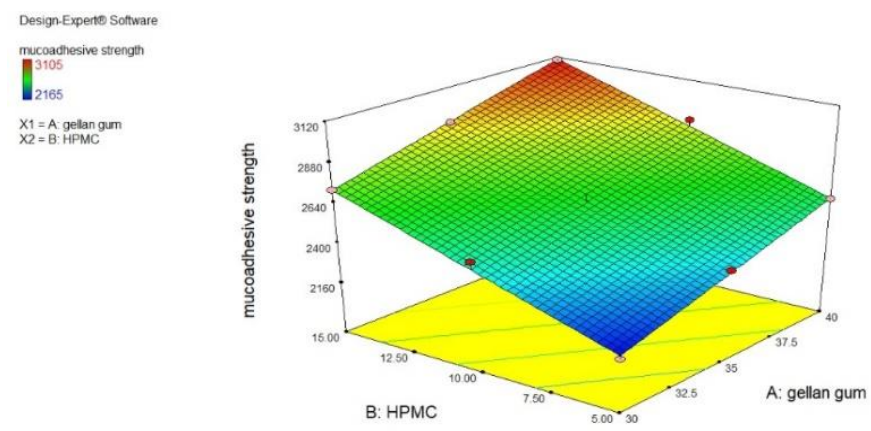

C) Counter plot of Mucoadhesive strength

Figure 1 Counter plots A) Counter plot of Drug release, B) Counter plot of Gel strength, and C) Counter plot of Mucoadhesive strength 


\subsection{Characterization of optimized RIZ loaded in-situ gel}

The gel strength of optimized ion activated in-situ gel was found to be 32.54 seconds. From the result, it was found that the Ion-activated in-situ gel showed suitable gel strength, which means that the gel would be easily retained in the nasal cavity. The gel strength values between 25 and $50 \mathrm{~s}$ are considered sufficient, as gel strength of less than $25 \mathrm{~s}$ may not preserve its integrity and may erode rapidly while gels with strength greater than $50 \mathrm{~s}$ are too stiff and may cause discomfort and may not release the drug.

The mucoadhesion force is an important parameter for in situ gelling nasal formulations, since it prolongs the nasal clearance of gels and increases its residence time in nasal cavity. The stronger the mucoadhesive force; more is the nasal residence time ${ }^{7}$. However, the strong mucoadhesion to nasal mucosal membrane might not actually be advantageous. The rapid mucus turnover is one of the most important protective mechanisms in the nose, and if the dosage form is extensively bound to the mucus it might be cleared from the mucosa at the same speed as that of the mucus ${ }^{8}$. The Mucoadhesive strength of the optimized batch of ion activated in-situ gel was found to be 2580.50 dynes $/ \mathrm{cm}^{2}$. Ideal value for the mucoadhesive strength is 2000 to 5000 dynes $/ \mathrm{cm}^{2}$ so the optimized batch was found to be in optimum range of mucoadhesive strength. The graph of mucoadhesive strength is depicted in figure 1.

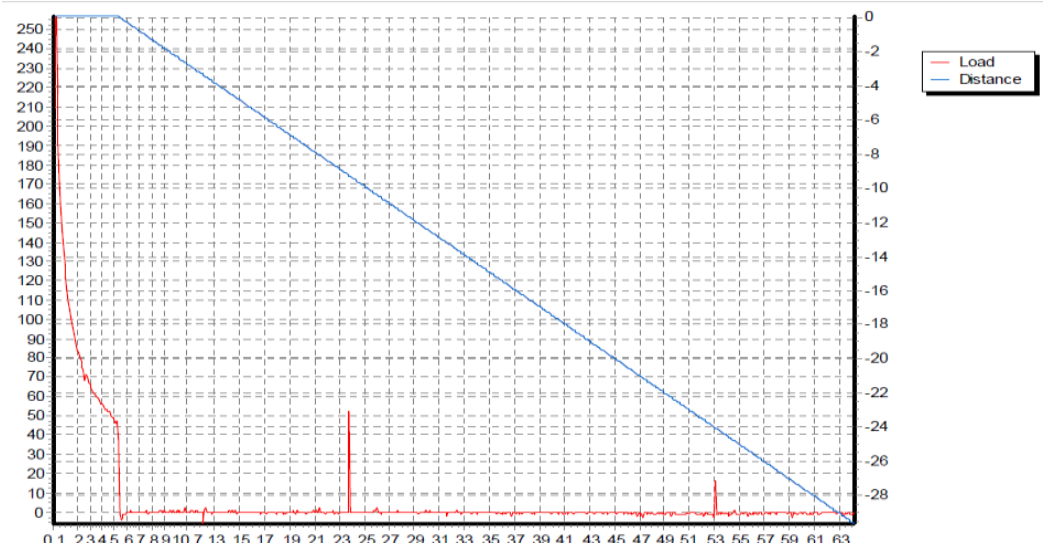

Figure 1: Mucoadhesive strength of Rizatriptan Benzoate in-situ gel

The drug content of the optimized batch of Ion activated in-situ gel was found to be $99.51 \pm 1.12 \%$. In-vitro release of RIZ from the plain drug solution and ion-activated gel is shown in figure 2 . It was observed that $94.82 \pm 2.15 \%$ drug was released from the plain drug solution within $3 \mathrm{~h}$, whereas $81.49 \pm 1.61 \%$ drug was released from ionactivated gel in $6 \mathrm{~h}$. Thus, it can be said that incorporation of RIZ in ion-activated gel could sustain the drug release in the gel matrix which act as reservoir from which the drug diffuses then finally gets diffused into the release medium.
The data obtained from the in-vitro drug release studies was fitted to the zero order, first order, higuchi model and korsmeyer- Peppas model. From the regression coefficient values of these models, the plot of Log \% CDR v/s log time i.e. korsmeyer- Peppas model for in-situ gel was found higher than others. Hence, we can conclude that the RIZ loaded in-situ gel korsmeyer- peppas model and the value of $\mathrm{n}$ (release exponent) was found to be 0.492 i.e. $0.45<\mathrm{n}<0.89$ indicates the anomalous diffusion/nonfickian diffusion.

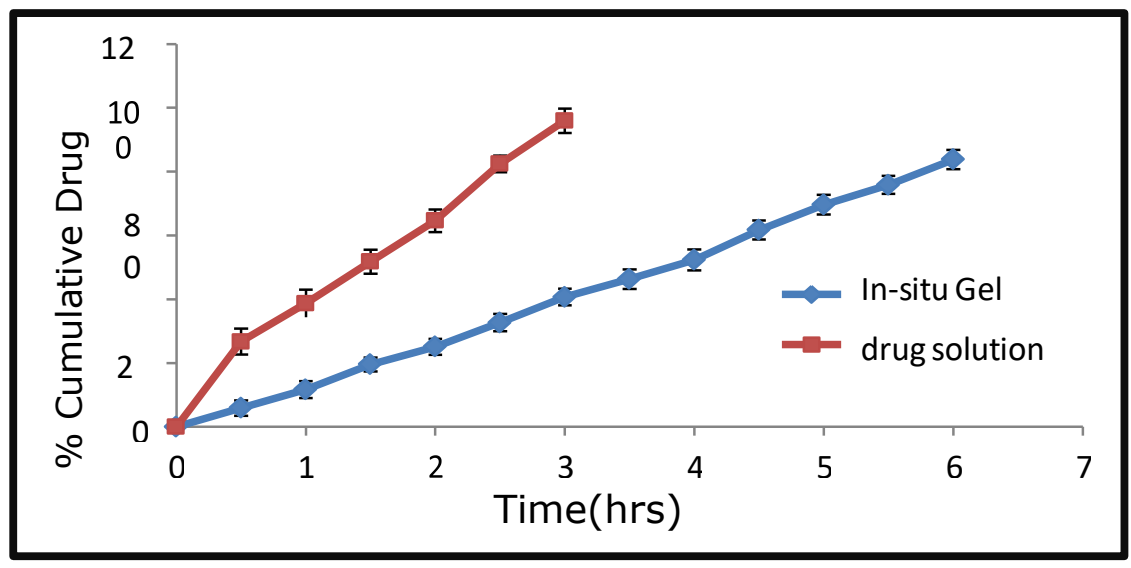

Figure 2: In-vitro release profile of optimized Rizatriptan Benzoate in-situ gel 
Ex-vivo drug permeation of RIZ from the plain drug solution and ion-activated gel is shown in figure 3. It was observed that only $40 \%$ drug was permeated from the plain drug solution, whereas more than $80 \%$ drug was permeated from ion-activated gel in $6 \mathrm{~h}$.

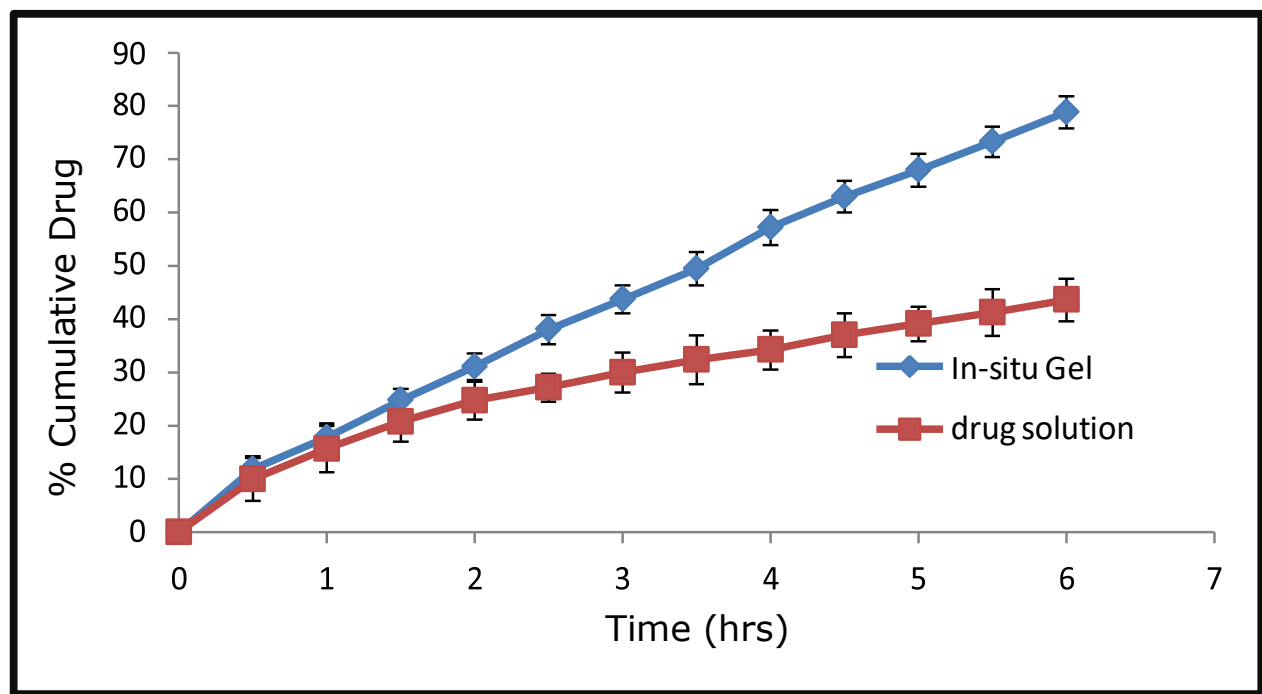

Figure 3: Ex-vivo drug permeation profile of optimized Rizatriptan Benzoate in-situ gel

The results of stability study at different temperature are shown in table 2. The stability of RIZ loaded Ion-activated Insitu gel was checked at room temperature $\left(25-40^{\circ} \mathrm{C}\right)$ and Refrigerated temperature $\left(2-8^{\circ} \mathrm{C}\right)$. The $\mathrm{pH}$ of the gel at refrigerated temperature was unchanged upto 2 months storage period. Gelation time of the gel did not show any major change at refrigerated temperature. Also, higher Drug Content of the gel was found when stored at refrigerated condition as compared to gel stored at room temperature. Hence, as compared to room temperature, refrigerated condition was found to be more preferable for the storage of RIZ loaded Ion-activated In-situ gel.

Table 2: Results of stability study of RIZ loaded in-situ gel

\begin{tabular}{|c|c|c|}
\hline & \multicolumn{2}{|c|}{ Storage condition for RIZ loaded Ion-activated in-situ gel } \\
\hline & Room temperature & Refrigerated temperature \\
\hline \multicolumn{3}{|c|}{ Physical Appearance } \\
\hline Initial & Clear and Transparent & Clear and Transparent \\
\hline After 15 days & Clear and Transparent & Clear and Transparent \\
\hline After 30 days & Clear and Transparent & Clear and Transparent \\
\hline After 60 days & Clear and Transparent & Clear and Transparent \\
\hline \multicolumn{3}{|c|}{ pH } \\
\hline Initial & $6.2 \pm 1.51$ & $6.2 \pm 1.12$ \\
\hline After 15 days & $6.1 \pm 1.21$ & $6.2 \pm 1.07$ \\
\hline After 30 days & $6.1 \pm 1.11$ & $6.1 \pm 0.99$ \\
\hline After 60 days & $6.0 \pm 1.31$ & $6.1 \pm 1.21$ \\
\hline \multicolumn{3}{|c|}{ Gelation time (sec) } \\
\hline Initial & $12 \pm 2$ sec & $11 \pm 2 \mathrm{sec}$ \\
\hline After 15 days & $12 \pm 2 \mathrm{sec}$ & $11 \pm 2 \mathrm{sec}$ \\
\hline After 30 days & $13 \pm 2 \mathrm{sec}$ & $11 \pm 3 \mathrm{sec}$ \\
\hline After 60 days & $14 \pm 2 \mathrm{sec}$ & $12 \pm 3 \mathrm{sec}$ \\
\hline \multicolumn{3}{|c|}{ Drug content ( $\%$ assay) } \\
\hline Initial & $99.49 \pm 2.12 \%$ & $99.49 \pm 2.12 \%$ \\
\hline After 15 days & $98.48 \pm 2.69 \%$ & $99.07 \pm 1.99 \%$ \\
\hline After 30 days & $97.59 \pm 1.99 \%$ & $98.70 \pm 1.24 \%$ \\
\hline After 60 days & $96.37 \pm 2.86 \%$ & $98.23 \pm 2.02 \%$ \\
\hline
\end{tabular}




\section{CONCLUSION}

In the present investigation, RIZ loaded ion activated in-situ gel was formulated and optimized using $3^{2}$ factorial design. It was further characterized for gelation time, drug content, gel strength, mucoadhesive strength and the iv-vitro studies supported the controlled drug release of RIZ loaded in-situ gel for prolonged period of time. The present investigation demonstrates that intranasal RIZ loaded in-situ gel can potentially transport RIZ to the brain and can serve as a noninvasive alternative for the delivery of RIZ to the brain.

Declaration of interest: The authors declare that they have no conflict interests.

\section{REFERENCES}

1. Javia, A., G. Kore, and A. Misra, Polymers in Nasal Drug Delivery: An Overview. Applications of Polymers in Drug Delivery, 2020: p. 305-332.

2. Ponkshe, P., et al., Nasal and Pulmonary Drug Delivery Systems, in In-Vitro and In-Vivo Tools in Drug Delivery Research for Optimum Clinical Outcomes. 2018, CRC Press. p. 79-134.

3. BEGLEY, D.J., The Blood-brain Barrier: Principles for Targeting Peptides and Drugs to the Central Nervous System. Journal of pharmacy and pharmacology, 1996; 48(2):136-146.

4. Jelkmann, M., et al., A gellan gum derivative as in-situ gelling cationic polymer for nasal drug delivery. International Journal of Biological Macromolecules, 2020.

5. Rupenthal, I.D., C.R. Green, and R.G. Alany, Comparison of ionactivated in situ gelling systems for ocular drug delivery. Part 1: physicochemical characterisation and in vitro release. International journal of pharmaceutics, 2011; 411(1-2):69-77.

6. Cao, S.-l., Q.-z. Zhang, and X.-g. Jiang, Preparation of ion-activated in situ gel systems of scopolamine hydrobromide and evaluation of its antimotion sickness efficacy. Acta Pharmacologica Sinica, 2007; 28(4):584-590.

7. Kaur, P., et al., In situ nasal gel drug delivery: A novel approach for brain targeting through the mucosal membrane. Artificial cells, nanomedicine, and biotechnology, 2016; 44(4):1167-1176.
8. Paul, A., K. Fathima, and S.C. Nair, Intra nasal in situ gelling system of lamotrigine using ion activated mucoadhesive polymer. The open medicinal chemistry journal, 2017; 11:222.

9. Ahmed, O.A. and S.M. Badr-Eldin, In situ misemgel as a multifunctional dual-absorption platform for nasal delivery of raloxifene hydrochloride: formulation, characterization, and in vivo performance. International Journal of Nanomedicine, 2018; 13:6325.

10. Menzel, C., et al., Nasal drug delivery: design of a novel mucoadhesive and in situ gelling polymer. International Journal of Pharmaceutics, 2017; 517(1-2):196-202.

11. Agrawal, M., et al., Stimuli-responsive In situ gelling system for nose-to-brain drug delivery. Journal of Controlled Release, 2020.

12. Sabale, A.S., A.D. Kulkarni, and A.S. Sabale, Nasal In Situ Gel: Novel Approach for Nasal Drug Delivery. Journal of Drug Delivery and Therapeutics, 2020; 10(2-s):183-197.

13. Vigani, B., et al., Recent Advances in the Development of In Situ Gelling Drug Delivery Systems for Non-Parenteral Administration Routes. Pharmaceutics, 2020; 12(9):859.

14. Yang, S.C., et al., Body distribution in mice of intravenously injected camptothecin solid lipid nanoparticles and targeting effect on brain. Journal of controlled release, 1999; 59(3):299307.

15. Alam, S., et al., Development and evaluation of thymoquinoneencapsulated chitosan nanoparticles for nose-to-brain targeting: a pharmacoscintigraphic study. International journal of nanomedicine, 2012; 7:5705.

16. Javia, A. and H. Thakkar, Intranasal delivery of tapentadol hydrochloride-loaded chitosan nanoparticles: formulation, characterisation and its in vivo evaluation. Journal of microencapsulation, 2017; 34(7):644-658.

17. Jang, K.-I. and H.G. Lee, Stability of chitosan nanoparticles for $L$ ascorbic acid during heat treatment in aqueous solution. Journal of agricultural and food chemistry, 2008; 56(6):1936-1941.

18. Guideline, I.H.T., Stability Testing Guidelines: Stability Testing of New Drug Substances and Products. ICH Q1A (R2)(CPMP/ICH/2736/99), 1999.

19. Ali, J., et al., Development and validation of a stability-indicating HPTLC method for analysis of antitubercular drugs. Acta Chromatographica, 2007; 18:168.

20. Branch, S.K., Guidelines from the international conference on harmonisation (ICH). Journal of pharmaceutical and biomedical analysis, 2005; 38(5):798-805. 